\title{
Image-based BRDF Representation
}

\author{
A. MIHÁLIK AND R. ĎURIKOVIČ
}

\begin{abstract}
:
To acquire a certain level of photorealism in computer graphics, it is necessary to analyze, how the materials scatter the incident light. In this work, we propose the method to direct rendering of isotropic bidirectional reflectance function (BRDF) from the small set of images. The image-based rendering is focused to synthesize as accurately as possible scenes composed of natural and artificial objects. The realistic image synthesis of BRDF data requires evaluation of radiance over the multiple directions of incident and scattered light from the surface. In our approach the images depict only the material reflectance, the shape is represented as the object geometry. We store the BRDF representation, acquired from the sample material, in a number of two-dimensional textures that contain images of spheres lit from the multiple directions. In order to render particular material, we interpolate between textures in the similar way the image morphing works. Our method allows the real-time rendering of tabulated BRDF data on low memory devices such as mobile phones.
\end{abstract}

Mathematics Subject Classification 2000: 78A05, 68P01, 68U05

Additional Key Words and Phrases: real-time rendering, gloss, BRDF

\section{INTRODUCTION AND RELATED WORK}

The appearance of all objects is determined by how object's surface scatters incident light [Tonsho et al. 2001]. Surfaces in general scatter incident light in different directions in consideration of the angles of incidence. Bidirectional Reflectance Distribution Functions (BRDFs) characterizes reflected light transport at an particular surface point.

Analytic reflection models provide the BRDFs used in computer graphics [Ashikhmin and Shirley 2000]. An example is the empirical Wards's model [Ward 1992], that uses the combination of functions capturing the reflection attributes such as the diffuse reflectance in all directions or the concentration of light scattering in a direction near the specular direction for glossy materials. Besides empirical models, models that apply basic principles of physics to the surface microscopic structure exist, for example the Cook-Torrance's model [Cook and Torrance 1982]. 
An alternative to directly measuring model parameters is to acquire actual samples from a BRDF using a gonio-spectrophotometer and then fit the measured data to a selected analytic model using various optimization techniques [Ďurikovivč and Mihálik 2014]. A gonio-spectrophotometer is an instrument that measures the spectral distribution of reflected radiant power as a function of angles of illumination and observation. The device consists of a light source aperture and a receptor aperture. The apertures are oriented in variety of directions to perform comprehensive measurements. A large database of tabular BRDF can be obtained by measurement of real materials [Matusik et al. 2003].

There are shortcomings to this measure-and-fit approach. A BRDF represented by the analytic function with the computed parameters is only an approximation of real reflectance. Measured values of the BRDF are usually not exactly equal to the values of the analytic model. Since most BRDF models are highly non-linear, the optimization frameworks used in the fitting process rely heavily on initial guesses of the models parameters.

Another approach is to acquire measurements of the BRDF and use these measurements directly as the sphere maps in the rendering pipeline represented by the shader tree [Ďurikovič et al. 2002].

There are some approaches to store the BRDF into the textures. Some exploit normalized matrix decomposition [Wynn 2001], others requires 3D textures [O’Toole 2007].

Our contribution is the method of image blending in order to obtain the BRDF of surface lit by the particular light source.

In our approach we use image based BRDF acquired from spherical samples. We utilize graphics processing unit (GPU) operating in parallel manner, that allow us to program the image synthesis using so called shader programs. In the rendering process we interpolate between actual images. We found out that few images as the input of shader program produce plausible results. Our representation allows us to 

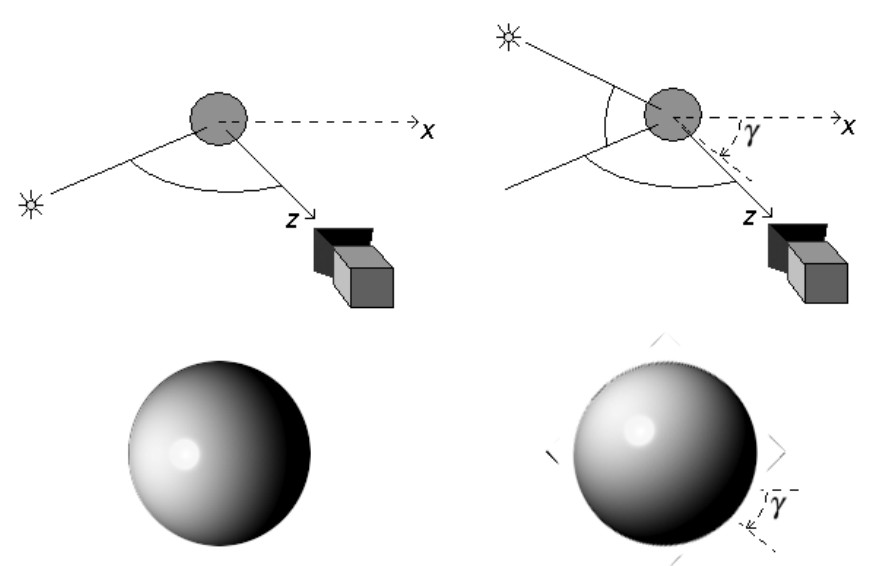

Fig. 1. The rotation of light source around the view direction yields the same result as the rotation of the image about the same angle.

render isotropic BRDF of real materials in real-time with less use of the memory than tabulated BRDF data representation.

The remaining of this paper is organized as follows. In Section 2 we describe the creation of textures used in the shader program. Section 3 discuss actual rendering from the set of image based BRDF textures. Finally we conclude our work in Section 5.

\section{IMAGE BASED BRDF REPRESENTATION}

If we consider the isotropic BRDF measurements, we are able to fix the viewing direction during the measuring process. This lose of one degree of freedom is usually compensated by the usage of spherical material samples. If we rotate light source around the viewing direction, we get the same result as if we fix the light source and rotate the camera around the viewing direction, see Figure 1. This leads us to the precondition that in the case of isotropic BRDF measurements of spherical samples, it is enough to rotate the light source just in the horizontal plane.

In our approach the images of unit sphere are stored in $2 \mathrm{D}$ textures. We use these textures as the input to our shader program that is running on the GPU. Each texture 


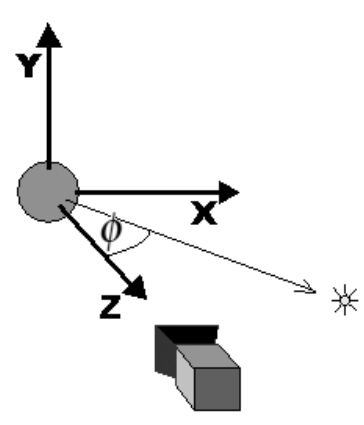

Fig. 2. Geometry of spherical sample rendering in order to acquire image based BRDF representation textures.

contains the unit sphere rendered under particular light source position using actual material BRDF. As we noted above, light source was positioned solely in the horizontal plane. To render the samples we have used the variety of isotropic BRDF tabular representations acquired from the MERL database of measured materials by [Matusik et al. 2003].

We tested materials like plastics, paints and metals such as aluminum or gold metallic paint. We render our BRDF representation according rendering equation using particular BRDF and single light source. Let us consider a given coordinate system, we place unit sphere in to the center of coordinate system. The view direction is along the negative $\mathrm{Z}$ axis and light direction forms particular angle $\phi$ with the $\mathrm{Z}$ axis in the plane $Y=0$, see Figure 2. We have acquired four images of the particular material sample under the set of light directions using $\phi=\left\{0^{\circ}, 45^{\circ}, 90^{\circ}, 135^{\circ}\right\}$. We found out that such low density of light direction sampling was working well in the case of plastics. In the case of metallics or the material surfaces that produce the color shift we used different light directions. Because they exhibit Fresnel effects in the specular reflection we decided to use slightly denser light direction sampling. We used angles $\phi=\left\{0^{\circ}, 30^{\circ}, 60^{\circ}, 90^{\circ}, 120^{\circ}, 150^{\circ}\right\}$. The result of rendered spherical samples is shown on Figure 3.

The images are then loaded into the video memory as the textures. We have used resolution $512 \times 512$ which we found suitable for general use. In the case of high 

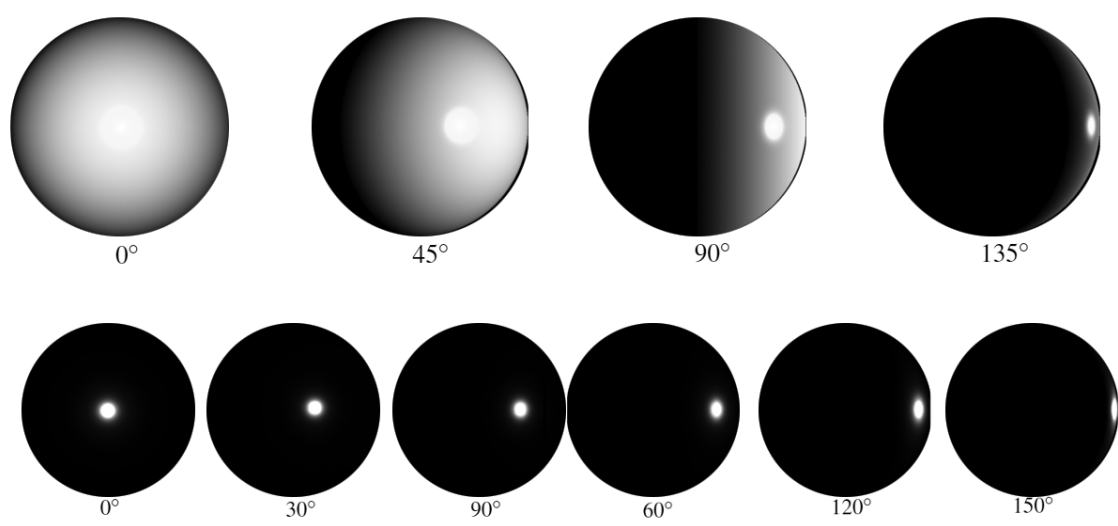

Fig. 3. Acquired image based BRDF textures under the particular light direction according to the angle $\phi$. First row represents yellow plastic sphere. Second row represents specular material that exhibits the notable color shift.

definition rendering we suggest to use the resolution at least $1024 \times 1024$. In our shader program we interpolate between the textures to render the material surface from the particular light source.

\section{IMAGE BASED BRDF SHADER}

In our shader program we use captured textures as an input. We have developed interpolation procedure in order to blend between the two images. Our approach was inspired by the image morphing techniques.

The input to the rendering algorithm is the set of image based BRDF textures, the light source and the object geometry. The light source direction is defined by the tuple of angles $(\gamma, \phi)$ according to the Figures 1 and 2. The angle $\gamma$ (azimuthal angle) refers to the rotation of the light source direction around the $\mathrm{Z}$ axis from the plane $Y=0$. The angle $\phi$ (polar angle) is an angle between the $\mathrm{Z}$ axis and the light direction. If we consider the object geometry, then in every surface point we have defined the normal vector perpendicular to the surface. In order to render the surface we rotate every normal vector about the angle $-\gamma$ to compensate for the rotation of the light direction around the $\mathrm{Z}$ axis. After the transformation we can consider the light source 
in the plane $Y=0$. Let represent the surface normal after this operation as the vector $\left(n_{x}, n_{y}, n_{z}\right)$. Note that the surface normals have to be transformed to the camera space. Then we can compute the texture coordinates and fetch the texels from our image based BRDF textures.

Now we will focus on the angle $\phi$. Let suppose the angle $\phi \in\left[0^{\circ}, 180^{\circ}\right]$, where $\phi_{1} \leq$ $\phi \leq \phi_{2}$. Angles $\phi_{1}$ and $\phi_{2}$ represent the angles of neighboring textures from the texture set of image based BRDF (Figure 3). Let $t_{\phi_{1}}(u, v)$ and $t_{\phi_{2}}(u, v)$ are the particular textures. To acquire the rendered image, we blend and interpolate between $t_{\phi_{1}}(u, v)$ and $t_{\phi_{2}}(u, v)$. We define angles and interpolation parameter $t$ as:

$$
\begin{gathered}
\Delta \phi_{1}=\phi_{1}-\phi, \\
\Delta \phi_{2}=\phi_{2}-\phi, \\
t=\frac{\phi-\phi_{1}}{\phi_{2}-\phi_{1}} .
\end{gathered}
$$

The final color of the surface point is computed as:

$$
\begin{array}{r}
I_{o}=I_{l} t_{\phi_{1}}\left(P_{z}\left(S\left(R_{y}\left(\Delta \phi_{1}, n_{x}, n_{y}, n_{z}\right)\right)\right)(1-t)+\right. \\
I_{l} t_{\phi_{2}}\left(P_{z}\left(S\left(R_{y}\left(\Delta \phi_{2}, n_{x}, n_{y}, n_{z}\right)\right)\right) t\right.
\end{array}
$$

where $I_{l}$ is the light intensity coefficient, $R_{y}(\alpha, x, y, z)$ is the rotation of vector $(x, y, z)$ around the $\mathrm{Y}$ axis about the angle $\alpha . P_{z}$ is the projection $P_{z}(x, y, z)=(x, y) . S$ is the function used in the spherical texture mapping $S(x, y, z)=\left(\frac{x}{2}+\frac{1}{2}, \frac{y}{2}+\frac{1}{2}, \frac{z}{2}+\frac{1}{2}\right)$. This yield us the result depicted in the Figure 4 a), which works well for the diffuse component. However, specular reflection is doubled around the point where the normal is equal to the halfway vector. Halfway vector is the vector halfway between the light source direction and the viewing direction. To fix this we can use halves of the angles $\Delta \phi_{1}$ and $\Delta \phi_{2}$ in Equation 2. However, this way we mess up the diffuse shading as we can see in Figure $4 \mathrm{~b}$ ). We should rotate the diffuse part about $\Delta \phi_{1}$ and $\Delta \phi_{2}$, respectively, while the specular part about $\frac{\Delta \phi_{1}}{2}$ and $\frac{\Delta \phi_{2}}{2}$, respectively. To do this we introduce the rotation coefficient $k$. For the points where the surface normal is close to the halfway vector, the coefficient $k$ should be close to $\frac{1}{2}$. Further from the 
halfway vector, $k$ should approach 1 . This is described by the following equation:

$$
\begin{array}{r}
k=\frac{1}{2}+\frac{\sin \alpha_{h}}{2} ; \\
\alpha_{h} \in\left[0, \frac{\pi}{2}\right],
\end{array}
$$

where $\alpha_{h}$ is the angle between the halfway vector and the vector $\left(n_{x}, 0, n_{z}\right)$. To render both diffuse and specular part appropriately, we multiply angles $\Delta \phi_{1}$ and $\Delta \phi_{2}$ in Equation 2 by the rotation coefficient $k$ in every surface point. Equation 2 can be rewritten:

$$
\begin{array}{r}
I_{o}=I_{l} t_{\phi_{1}}\left(P_{z}\left(S\left(R_{y}\left(k \Delta \phi_{1}, n_{x}, n_{y}, n_{z}\right)\right)\right)(1-t)+\right. \\
I_{l} t_{\phi_{2}}\left(P_{z}\left(S\left(R_{y}\left(k \Delta \phi_{2}, n_{x}, n_{y}, n_{z}\right)\right)\right) t .\right.
\end{array}
$$

This leads to the computation of texture coordinates to fetch the texels from the specular regions of both textures $t_{\phi_{1}}(u, v)$ and $t_{\phi_{2}}(u, v)$. Then it blends the particular texels according to the interpolation parameter $t$ (Equation 1) to acquire the colors in the region where the surface normal approaches the halfway vector. In other regions the computation of texture coordinates is based on the rotation that is the approximation of rotation about the angle $\Delta \phi_{1}$ and $\Delta \phi_{2}$, respectively.

If the angle $\phi$ exceed greatest light direction angle of image based BRDF textures, then $\phi_{1} \geq 135^{\circ}$. In this case we map only the texture $t_{\phi_{1}}(u, v)$ with the rotation coefficient $k=\frac{3}{4}$ in the texture coordinate computation.

\section{RESULTS}

We use the trilinear texture filtering in our experiments. Depending on the number of light sources we have two approaches of accessing the textures in our shader program. If we consider only the one light source we can access just two textures from the shader program. Then we use two texture units. According the light source position we choose textures $t_{\phi_{1}}(u, v)$ and $t_{\phi_{2}}(u, v)$ and bind them to the particular texture unit on the CPU. In the case of multiple light sources, skydome or possibly importance sampling and if the GPU supports enough number of texture units, we should bind every texture from the set of image based BRDF textures (Figure 3) into separate 


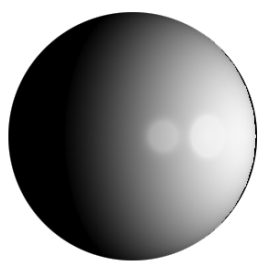

a)

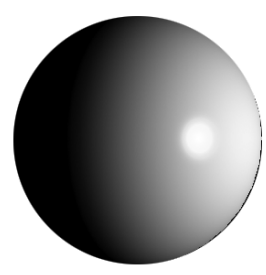

c)

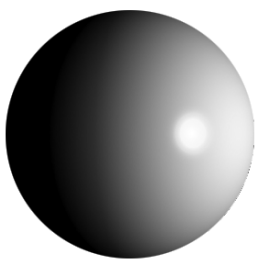

b)

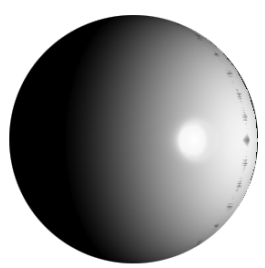

d)

Fig. 4. Rendered image of sphere where $\phi=60^{\circ}, \phi_{1}=45^{\circ}, \phi_{2}=90^{\circ}$. a) $\Delta \phi_{1}=-15^{\circ}, \Delta \phi_{2}=55^{\circ}$. b) In Equation 2 we used $\frac{\Delta \phi_{1}}{2}$ instead of $\Delta \phi_{1}$ and $\frac{\Delta \phi_{2}}{2}$ instead of $\Delta \phi_{2}$, respectively. Note the sharper band in the diffuse shade. c) The desired result of image based BRDF rendering technique with correct diffuse and specular component according to Equation 4. d) Notable artefacts produced by the texture filtering.

texture unit on the GPU. Then we can decide which texture to use while processing particular light direction in the shader program.

During our experiments we encountered the problem when resulting texture coordinates exceeded the boundaries of the sphere in the texture image. This is caused by the texture filtering which led to the artefacts depicted in Figure 4 d). To solve this problem, we simply shorten the length of surface normal by the multiplication of normal by the factor 0.995 .

In Figure 5 are depicted results of multiple materials applied to the actual objects using the directional light source.

We tested our approach on GPU ATI Mobility Radeon HD 4650. Table I describes rendering performance using multiple light sources. Since our method is based mainly on the texture fetch, addition of extra light source does not significantly decrease the performance. Resulting image using 4 light sources is depicted in Figure 6. 


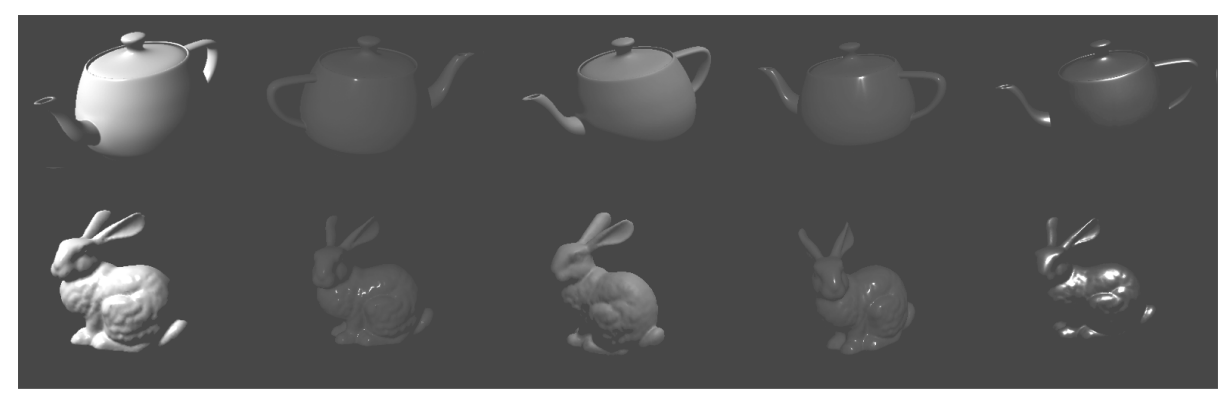

a)

b)

c)

d)

e)

Fig. 5. Rendered results. a) Yellow plastics. b) Red specular plastics. c) Orange diffuse paint. d) Blue acrylic. e) Aluminum bronze.

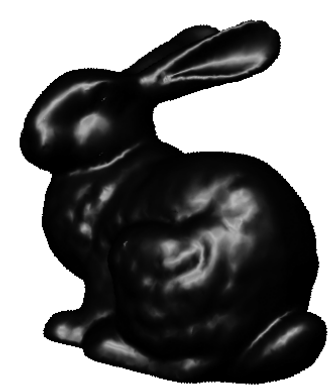

Fig. 6. Rendered image of gold BRDF using 4 point lights.

\section{CONCLUSIONS}

Under the assumption of isotropic opaque surface we have developed the procedure to real-time rendering of arbitrary BRDF. The procedure utilizes few images created from the measured BRDF of real materials. This approach is an alternative to the tabulated BRDF with less memory consumption, which makes it suitable for the embedded devices such as mobile phones.

\begin{tabular}{|l|l|l|l|l|}
\hline no of lights & 1 & 2 & 4 & 8 \\
\hline bunny & $325 \mathrm{fps}$ & $324 \mathrm{fps}$ & $324 \mathrm{fps}$ & $323 \mathrm{fps}$ \\
\hline teapot & $388 \mathrm{fps}$ & 384fps & 383fps & 380fps \\
\hline
\end{tabular}

Table I. Frames per second according to the 3D model and the number of light sources. 


\title{
REFERENCES
}

Ashikhmin, M. And ShiRley, P. 2000. An anisotropic phong brdf model. J. Graph. Tools 5, 2 (Feb.), 25-32.

Cook, R. L. And TorRance, K. E. 1982. A reflectance model for computer graphics. ACM Trans. Graph. 1, 1, 7-24.

Ďurikovič, R., Kolchin, K., AND ERShOv, S. 2002. Rendering of japanese artcraft. In Short Presentations of EUROGRAPHICS Conference. Braunschweig, Germany, 131-138.

Matusik, W., Pfister, H., Brand, M., And McMillan, L. 2003. A data-driven reflectance model. In SIGGRAPH '03: ACM SIGGRAPH 2003 Papers. ACM, New York, NY, USA, 759-769.

O'TOOLE, M. 2007. Real-time rendering of acquired BRDF data sets.

Tonsho, K., Akao, Y., Tsumura, N., And MiYake, Y. 2001. Development of goniophotometric imaging system for recording reflectance spectra of 3D objects. In Society of Photo-Optical Instrumentation Engineers (SPIE) Conference Series, R. Eschbach and G. G. Marcu, Eds. Society of Photo-Optical Instrumentation Engineers (SPIE) Conference Series, vol. 4663. 370-378.

ĎURIKOVIVČ, R. AND MIHÁLIK, A. 2014. Modeling the BRDF from spectral reflectance measurements of metallic surfaces. Applied Surface Science 312, 0, 87 - 90.

WARD, G. J. 1992. Measuring and modeling anisotropic reflection. SIGGRAPH Comput. Graph. 26, 2, 265-272.

WYNN, C. 2001. Real-time brdf-based lighting using cube-maps. Available from http://www.nvidia.com/.

\author{
Andrej Mihálik \\ Mathematics, Physics and Informatics, \\ Comenius University, \\ 84248 Bratislava, Slovak Republic \\ http://www.fmph.uniba.sk \\ email: mihalik@sccg.sk \\ Roman Ďurikovič \\ Faculty of Mathematics, Physics and Informatics, \\ Comenius University, \\ 84248 Bratislava, Slovak Republic \\ http://www.fmph.uniba.sk \\ email: roman.durikovic@fmph.uniba.sk
}

